\title{
Téoros
}

Revue de recherche en tourisme

\section{La villégiature : un phénomène important}

\section{Marcel Samson}

Volume 7, numéro 2, juillet 1988

Villégiature et tourisme

URI : https://id.erudit.org/iderudit/1080407ar

DOI : https://doi.org/10.7202/1080407ar

Aller au sommaire du numéro

Éditeur(s)

Université du Québec à Montréal

ISSN

0712-8657 (imprimé)

1923-2705 (numérique)

Découvrir la revue

Citer ce document

Samson, M. (1988). La villégiature : un phénomène important. Téoros, 7(2), 1-2. https://doi.org/10.7202/1080407ar d'utilisation que vous pouvez consulter en ligne.

https://apropos.erudit.org/fr/usagers/politique-dutilisation/ 
Avec l'effet catalyseur du tourisme créé par les centres de villégiature, la popularité des stations de ski et conséquemment la multiplication de la résidence secondaire et sa transformation de plus en plus frequente en résidence toute saison, des promoteurs immobiliers professionnels sont apparus sur le terrain. Cela a définitivement consacré les espaces de villégiature les plus recherchés (distance-temps, services, activités hiver-été...) dans le mode d'exploitation urbain. Ce sont des acteurs économiques venus pour la plupart de l'extérieur; les lieux de décision économique ont donc échappé le plus souvent aux populations locales. Une population locale qui d'ailleurs a un mode de vie façonné sur la culture urbaine et qui laissera, dans la foulée des mouvements conservationnistes, la protection de son patrimoine ou de son cadre de vie dans les mains des résidents secondaires.

Ces nouveaux promoteurs feront surgir du paysage des nouveaux développements non riverains du type "villages de vacances" ou on retrouvera des équipements de loisir propres à une clientèle privilégiée (plage, piscine, tennis, piste de ski de randonnée, centre communautaire, ...) calqués sur le style nouvelle banlieue; ces villages ont l'avantage de fournir un cadre de vie recherché et d'assurer, en prime, l'intimité du propriétaire.

Enfin, I'immeuble en copropriété, est le dernier concept apparu depuis les cinq dernières années dans les sites où la spéculation immobilière est la plus féroce. On a donc bouclé la boucle de la forme de I'habitat urbain en milieu de villégiature. Localisés sur les rives des plans d'eau les plus cotés ou aux pieds des pentes de stations de ski les plus "fashionables", ces logements collectifs permettent de rentabiliser l'espace au maximum et jusqu'à présent ont surtout été érigés dans un but locatif. Ainsi, le comportement de consommateurs de résidences secondaires comme le mode d'implantation de I'habitat lui-même relèvent ces dernières années d'un schéma proprement urbain.

Comment cette évolution va-t-elle se poursuivre? Avec phus de cent cinquante mille résidences secondaires dans les régions limitrophes de l'agglomération montréalaise et quelques milliers d'autres d'ailleurs, le Québec détient avec I'Ontario le record canadien. C'est aussi un des p/us hauts taux en Amérique du Nord. Pourtant, sous le coup de la conjoncture associée à d'autres facteurs, le marché du chalet à la campagne a semblé s'effondrer ces dernières années. Est-ce vraiment la fin d'un rêve pour nombreux citadins?

Après plus de vingt ans d'engovement pour un chalet "les pieds dans l'eau". I'euphorie semble bel et bien finie pour beaucoup de ménages. La "popularisation" de la résidence secondaire est maintenant chose du passé. Après la grande villégiature du siècle dernier, la proliferation des petits chalets saisonniers de l'après-deuxième 
guerre mondiale, voilà que nous sommes revenus à une sorte d'appropriation exclusive de l'habitat de loisir de proximité par des ménages plus privilégiés. I/ reste cependant que la résidence secondaire demeure toujours un symbole et un signe de reconnaissance au sein des catégories socio-économiques de la société. Ainsi, une lecture superficielle des changements socio-économiques pourrait laisser croire que les jeunes ménages d'aujourd'hui versent dans l'hédonisme, que l'écologie verte s'estompe, que le mythe de la nature s'étiole... On s'adonne plutôt à la culture physique et au culte de sa nature. On serait désormais "poly-sensuel", on profiterait de la vie plutôt que d"amasser un aléatoire patrimoine pour demain. De plus, les agences de voyage seraient de redoutables concurrents: tous sont saisis de la bougeotte, jeunes comme retraités. Bref, la cote d'amour de la résidence secondaire aurait nettement baissé.

Ce type de discours a un certain sens dans la mesure où la résidence secondaire, comme pour la deuxième voiture, durant les premières années de la croissance économique d'après-guerre est vite devenue un nec-plusu/tra de la réussite sociale; un bien de consommation passager en quelque sorte. Dans la mesure aussi où cette résidence secondaire se localise dans un espace de villégiature qui n'a rien à voir avec l'espace rural de proximité de l'agglomération urbaine. Cependant, la résidence secondaire s'est développée historiquement dans l'unité du système urbain/péri-urbain/rural. Aussi, les contextes démographiques et économiques d'ensemble dans lesquels se sont effectuées les grandes vagues de rurbanisation restent fondamentaux. Ce sont ces dernières qui permettent d'expliquer d'abord les mutations de l'espace rural de proximité mais aussi, et de façon très nette, les vagues de migration hebdomadaire ou saisonnière dans un espace rural qui n'est pas comme les autres puisque c'est avant tout un espace de villégiature.

Les textes que nous avons réunis dans ce numéro de Téoros sur la villégiature illustrent de façon très claire les propos que nous venons de tenir. Nous avons cependant tenu à élargir le concept non pas seulement à la seule résidence secondaire mais aussi à d'autres formes, comme la pourvoirie par exemple. Nous avons de plus voulu exprimer des facettes moins positives de la villégiature privée, comme ses effets sur l'environnement naturel. L'ensemble des aspects abordés dans le présent numéro ne permettent pas de faire le tour de la question mais soulevent suffisamment de questions pour que les différents intervenants à quelque palier que ce soit se sentent concernés par ce phénomèneilat $f$

Notes explicatives

(1) RAGATZ, Richard Lee (1970), "Vacation Housing: A Missing Component in Urban and Regianal Theory", Land Economics, Vol. 6, No 2, pp. 118-126.

(2) ST-AMOUR, Jean-Pierre (1979), La villégiature au Quêbec, Hull, Ed. Asticou, $178 \mathrm{p}$.

(3) Nous tenons à remercier particulierement notre collague Roger Nadeau pour son assistance à l'álaboration de ce numéro.

\section{À la mémoire de Francine McKenzie}

Madame Francine Mckenzie, présidente du Conseil du statut de la femme, est décédée récemment. Le Québec a perdu en elle une grande humaniste. Téoros veut faire écho à sa lutte pour que les 200000 Haïtiens qui coupent la canne à sucre dans les 240 " "bateyes" de la République Dominicaine retrouvent leur dignité humaine. Contraire à la Déclaration Universelle des Droits de l'Homme, cette situation de quasi-esclavage ne peut durer; il faut boycotter la République Dominicaine comme destinationsoleil.

\section{N.D.L.R.}

